\title{
Analysis of Driver Drowsiness Detection System by using Soft Computing
}

\author{
Sapna Bhagat \\ M.Tech Scholar Department ECE \\ Amritsar College of Engineering \& Technology \\ Amritsar, India
}

\author{
V. K. Banga, PhD \\ Prof.ECE \& Principal \\ Department ECE \\ Amritsar College of Engineering \& Technology \\ Amritsar, India
}

\begin{abstract}
This report presents an automatic drowsy driver recognition and accident prevention system that is based on facial expressions changes. The key reason of traffic incidents could be because of drowsiness due to the number of years driving. Examining the facial expression can provide offer the prediction of driver's drowsiness to create the caution for the driver. Therefore, this report presents the drowsiness recognition approach for applying in vehicles. Our approach is accomplished by having a driver's facial image, searching the facial characteristics by image handling and analyzing the driver's drowsiness stage by utilizing hybrid technique.
\end{abstract}

\section{Keywords}

Driver Fatigue Recognition, Smart Vehicles, Facia appearance recognition, face detection, Neuro-Fuzzy, genetic.

\section{INTRODUCTION}

The word "drowsiness" is employed here to refer to their state of reduced alertness, frequently associated with performance and psychophysiological improvements that may bring about lack of alertness. The word "driver somnolence" is widely used to explain this disorder, especially on police incident reports and in incident data files [1].Many of deaths could possibly be eliminated if driver drowsiness was precisely monitored and individuals were given early warnings. Driver drowsiness, which is a type of excessive sleepiness, occurs when people are driving for extended periods of time in monotonous environments, such as for instance highways [2].

Many studies have been conducted on driver drowsiness detection. Yan Zhang et al. [3] detected driver drowsiness by using SVM with Boost-LBP features to analyze the facial expression images. Arun Sahayadhas et al. [4] focused on developing a system that can detect hypo vigilance, which includes equally drowsiness and inattention applying Electrocardiogram (ECG) and Electromyogram (EMG) signals. ECG and EMG signals combined with the video recording have already been gathered through the entire experiment. Chih-Jer Lin et al. [5] detected driver drowsiness by using embedded system which wasn't developed in past. The biomedical signal handling have already been applying to solve biochemical sciences issues, such as for example mindbrain imaging technology, examining cranial nerves productive dynamic distributed and further brain information processing mechanism. Leng, Lee Boon et al. [6] driver drowsiness detection system had been developed as mobile device application such as Percentage of Eye Closure (PERCLOS) measured by using mobile device camera. Gang $\mathrm{Li}$ et al. [7] used wearable EEG process which is made up of a Bluetooth-enabled EEG headband and a commercial smart watch was applied to evaluate the model in a real-time way.
The previous system only concentrate on either bio-signal or driver behavior with single methods. Our system uses combined technique to make the detection system more accurate.

\section{EXPERIMENT}

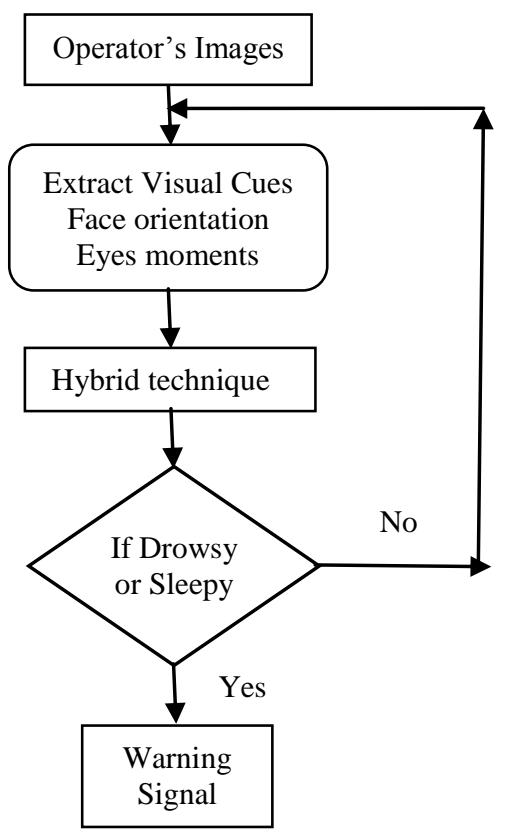

Fig 1: A flowchart of the proposed vigilance monitoring system

\subsection{Image Acquisition System}

Image understanding of visible behaviors starts with picture acquisition. The picture acquiring techniques have already been significantly recognized and applied. The objective of picture acquisition is to acquire the consecutive facial images of the driver face in real time. There is a camcorder is used to take the video of the driver and change into image frames. The size of the image should be $92 * 112$ pixels.

\subsection{Image Processing And Extract Visual Cues}

Image processing is to image analyses the extraction of meaningful information from images and handling of images applying mathematical operations by utilizing any form of signal processing and image ROI extraction (face orientation and eyes moment) then detect the facial expression by using hybrid method. 


\subsection{Hybrid Technique To Detect Drowsiness}

Neural system architectures are inspired by types of our personal minds and nerve cells. [1] As an example, a neural system for handwriting acceptance is described some feedback neurons which might be triggered by the pixels of an input image. [4] Similarly a neural network based algorithms are determined the amount of fatigue by measuring the facial expressions of driver accordingly. There are different learning methods are used to detect the fatigue like supervised, unsupervised and reinforcement learning. For example, itenderpal singh et al. [6] used the viola jones algorithm to detect the face which discovering the feedback picture method of sub screen effective at finding features.

The idea of unclear collection is just a type with unsharp boundaries. It offers a cause for basis for a qualitative method of the evaluation of complicated methods where linguistic as opposed to statistical factors are applied to describe program behaviour and efficiency. Fatigue is an application of fuzzy physical state.it cannot be quantified fairly so, they used pcs to utilize the fuzzy reasoning and establish the degree of fatigueness. [8] The facial characteristics such as for example vision and mouth opening, they need to fuzzily the values of those characteristics for the fuzzy sensation inference. For reaching this goal, the dimensions that they calculated for Mouth Opening, vision Opening, and Eyebrow Constriction are protected into five distinctive fuzzy units: very low, low, moderate, high, and very high; and dimensions acquired on Mouth edges Displacement is protected into three distinctive fuzzy units: LOW, MODERATE, and HIGH. And eventually, every secondary function is protected into two fuzzy units: LOW and HIGH [12].

\subsection{Performance Evaluation}

This paper has developed and executed planned method in MATLAB software R2013a. The planned algorithm is extremely a lot better than present practices. A contrast is drawn between most of variables and particular tables and figures display most of results.

We have the acquired images which we choose from the video, Images like normal, drowsy, sleepy. Partial drowsy, partial sleepy etc.

\section{If value of the image $<20$ \\ Results are Norma}

If value of the image $<20<40$

Results are Drowsy and generate alert alarm

If value of the image $<40<100$

Results are Sleepy and warning alarm is generate

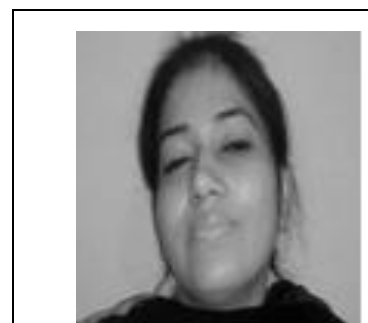

Normal image

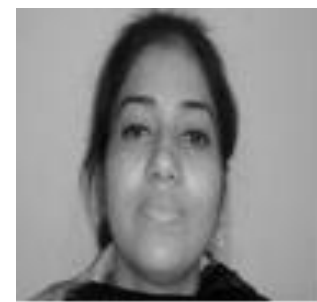

Drowsy image

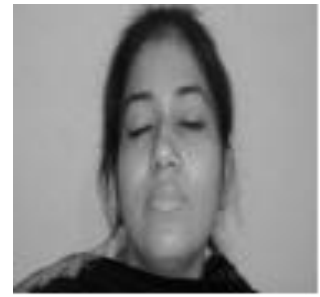

Sleepy image

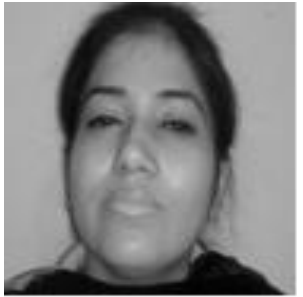

Partial drowsy image

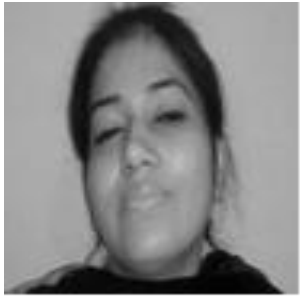

Partial sleepy image

Fig 1: Results of Proposed Technique

Table 1

\begin{tabular}{|c|c|c|l|}
\hline Image & Normal & Drowsy & Sleepy \\
\hline Normal & 70.2244 & 14.7673 & 15.0083 \\
\hline Drowsy & 10.4035 & 74.8884 & 14.7082 \\
\hline Sleepy & 15.7387 & 5.6791 & 78.5822 \\
\hline $\begin{array}{c}\text { Partial } \\
\text { Drowsy }\end{array}$ & 29.3059 & 41.5110 & 29.1831 \\
\hline Partial Sleepy & 22.3681 & 21.7748 & 55.8571 \\
\hline \multicolumn{2}{|c|}{} & & \\
\hline
\end{tabular}

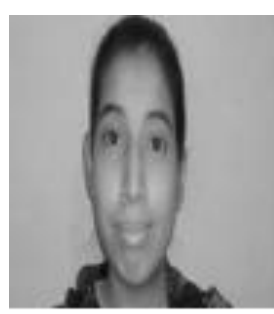

Normal image

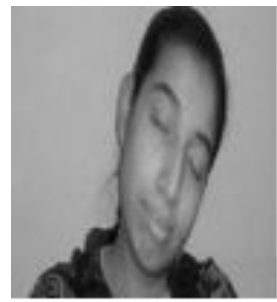

Sleepy image

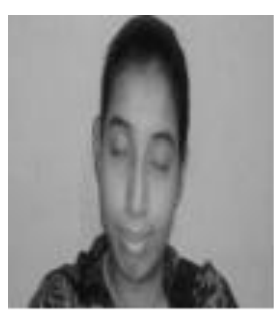

Drowsy image

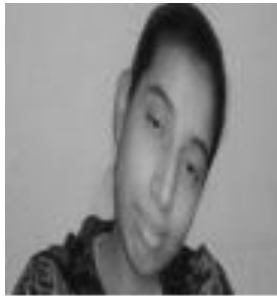

Partial Drowsy image 


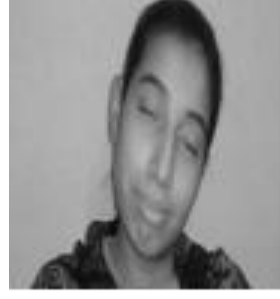

Partial Sleepy image

Fig 1: Results of Proposed Technique

Table 2

\begin{tabular}{|c|c|c|c|}
\hline Image & Normal & Drowsy & Sleepy \\
\hline Normal & 82.3544 & 5.4291 & 12.2164 \\
\hline Drowsy & 13.6820 & 76.4508 & 9.8672 \\
\hline Sleepy & 14.9530 & 9.9217 & 75.1253 \\
\hline $\begin{array}{c}\text { Partial } \\
\text { Drowsy }\end{array}$ & 20.5210 & 52.7409 & 26.7382 \\
\hline Partial Sleepy & 30.7996 & 23.3330 & 45.8674 \\
\hline \multicolumn{3}{|l}{}
\end{tabular}

\section{CONCLUSION}

In this report, we have examined the existing state of the information about driver inattention monitoring. Driver inattention increases driving risk and has changed into a significant aspect in a considerable percentage of traffic accidents. However, centred on a review of the literature, we classify driver inattention into two main categoriesdistraction and fatigue. The proposed system detects the driver drowsiness level based on facial expressions which are recognized by using neural networks and fuzzy logic. By applying the hybrid technique system gets the accurate results.

\section{REFERENCES}

[1] Sonali Rajput and Dr. V. K. Banga "Detecting Driver Somnolence Using Artificial Approach" 2 nd International Conference on Computer and Intelligent Systems (ICCIS'2014) \& 2 nd International Conference of Electrical, Electronics, Instrumentation and Biomedical Engineering (ICEEIB'2014) Bangkok(Thailand) 30-31 May, 2014,pp.31-34.

[2] Gang Li Boon-Leng Lee; Wan-Young Chung "Smartwatch-Based Wearable EEG System for Driver Drowsiness Detection Sensors" IEEE Vol.15 2 Sep, 2015, pp.7169- 7180 .

[3] Yan Zhang, Caijian Hua “ Driver fatigue recognition based on facial expression analysis using local binary patterns" Optik - International Journal for Light and Electron Optics Vol.126(23),Dec-2015,pp.4501-4505.

[4] Yunhua Chen, Weijian Liu, Ling Zhang, Mingyu Yan, Yanjun Zeng "Hybrid facial image feature extraction and recognition for non-invasive chronic fatigue syndrome diagnosis" computers in biology and medicine, $V o l .64,1$

[5] Kwok Tai Chui Kim Fung Tsang; Hao Ran Chi; Chung $\mathrm{Kit} \mathrm{Wu}$ "Electrocardiogram based classifier for driver drowsiness detection Industrial Informatics (INDIN)" IEEE 13th International Conference on 22-24 July, 2015 pp. $600-603$.

[6] Hachisuka, S "Human and Vehicle-Driver Drowsiness Detection by Facial Expression" Biometrics and Kansei Engineering (ICBAKE), International Conference on 5-7 July, 2013, pp.320 - 326 .

[7] Itenderpal Singh, Prof. V.K.Banga "Development of a Drowsiness Warning System Using Neural Network" International Journal of Advanced Research in Electrical, Electronics and Instrumentation Engineering Vol.2 (8), Aug-2013, pp.3614-3623.

[8] Takehiro Yamakoshi' Kenta,Matsumura, Peter Rolfe "Controlled mechanical vibration applied to driver's right heel to sustain alertness: Effects on cardiovascular behavior" Transportation Research Part C: Emerging Technologies Vol.38, Jan-2014, pp.101-109.

[9] Nidhi Sharma Prof. V. K. Banga "Development of a Drowsiness Warning System based on the Fuzzy Logic" International Journal of Computer Applications (0975 8887) Vol.8, 9 Oct, 2010.pp.1-6.

[10] Leng, Lee Boon Giin, LeeBoon; Chung, Wan-Young "Wearable driver drowsiness detection system based on biomedical and motion sensors" IEEE 1-4 Nov, 2015 pp. $1-4$.

[11] M.Patel, S.K.L. Lal, D. Kavanagh, P. Rossiter "Applying neural network analysis on heart rate variability data to asses' driver fatigue" Expert systems with applications, Vol.38,6 June , 2011, pp.7235-7242.

[12] Mahdi Ilbeygi , HamedShah-Hosseini "A novel fuzzy facial expression recognition system based on facial feature extraction from color face images" Engineering Applications of Artificial Intelligence, Vol.25(1), Feb 2012,pp. 130-146.

[13] N.G.Narole, P.R.Bajaj “A Neuro-Genetic System Design for Monitoring Driver's Fatigue: A Design Approach" IJSSST, Vol.10, pp.1-6.

[14] Maneesha V Ramesh, Aswathy K. Nair Abishek Thekkeyil Kunnath "Real-Time Automated Multiplexed Sensor System for Driver Drowsiness Detection" IEEE conference on 23-25 sept, 2011, pp.1-4.

[15] Wei Zhang, Youmei Zhang, Lin Ma, Jingwei Guan, Shije "Multimodal learning for facial expression recognition" Recognition Vol 48, Oct 2015, pp.31913202 .

[16] M.Magno, C.spagnol, L.Benini, E.Popovici "A low power wireless node for contact and contactless heart monitoring" Microelectronics journal, Vol.45, 2014, pp.1656-1664.

[17] Hachisuka, S "Human and Vehicle-Driver Drowsiness Detection by Facial Expression "Biometrics 5-7 July, 2013, pp.320- 326. 